\title{
The role of collateral disease theory in the prevention and treatment of atherosclerosis in post-menopausal women: a narrative review
}

\author{
Dan Huang ${ }^{1,2,3}$, Zhenhua Jia ${ }^{3}$, Liping Chang ${ }^{3}$, Hongru Zhou ${ }^{3}$, Xuan Wu ${ }^{3}$, Cong Wei ${ }^{3}$ \\ ${ }^{1}$ Affiliated Hospital of Integrated Traditional Chinese and Western Medicine, Nanjing University of Chinese Medicine, Nanjing, China; ${ }^{2}$ Jiangsu \\ Province Academy of Traditional Chinese Medicine, Nanjing, China; ${ }^{3}$ National Key Laboratory of Collateral Disease Research and Innovative \\ Chinese Medicine, Shijiazhuang, China \\ Contributions: (I) Conception and design: C Wei, D Huang; (II) Administrative support: C Wei; (III) Provision of study materials or patients: Z Jia, L \\ Chang; (IV) Collection and assembly of data: D Huang, H Zhou, X Wu; (V) Data analysis and interpretation: D Huang; (VI) Manuscript writing: All \\ authors; (VII) Final approval of manuscript: All authors. \\ Correspondence to: Cong Wei. National Key Laboratory of Collateral Disease Research and Innovative Chinese Medicine, Shijiazhuang 050035, \\ China. Email: weicong@yiling.cn.
}

\begin{abstract}
There was no equivalent term as "atherosclerosis in postmenopausal women" in ancient Chinese medical literature, so there was no precise method to treat this disease. The collateral disease theory is a theoretical system with the characteristics of traditional Chinese medicine, which studies two branches: qi collateral theory and vessel collateral theory. We first analyzed the relationship between qi and vessel collaterals. The collaterals are divided into the qi collaterals of circulating qi and the vessel collaterals of circulating blood. Qi collaterals and vessel collaterals play the role of circulating qi and blood together. We then illustrate that the concept of vessel collateral system in traditional Chinese medicine is like the concept of the vascular system in modern medicine, and atherosclerosis (AS) is a common vessel collateral-vascular system disease. A significant increase in the incidence of AS in postmenopausal women is related to estrogen deficiency, associated with dysfunction of the qi collaterals. AS in postmenopausal women is associated with both qi collaterals and vessel collaterals. Bazi Bushen capsule (BZBS) under the guidance of collateral disease theory replenishes kidney essence, coordinates yin and yang, tonifies kidney qi, and circulates blood. Meanwhile, it has 11 unique phytoestrogens (PEs), which resists AS. In this work, for the first time, we combined the vessel collateral theory with qi collateral theory. We explore the potential theoretical mechanism of the prevention and treatment of postmenopausal AS by the BZBS under the guidance of collateral disease theory.
\end{abstract}

Keywords: Collateral disease theory; vessel collateral theory; qi collateral theory; atherosclerosis after menopause (AS after menopause); Bazi Bushen capsule (BZBS)

Submitted May 21, 2020. Accepted for publication Jul 09, 2020.

doi: 10.21037/apm-20-1257

View this article at: http://dx.doi.org/10.21037/apm-20-1257

\section{Background}

Atherosclerosis (AS), a chronic, systemic inflammation manifesting in blood vessels, is the primary origin of coronary artery disease and stroke AS can lead to cardiocerebrovascular endpoint events in both men and women $(1,2)$. Thanks to the protective effects of estrogen on the cardiovascular system (3), the incidence of coronary artery disease in women before menopause are 1/10-3/10 of that in men (4); however, the risk increases ten times after menopause (5). Menopause is a key risk factor for developing coronary artery disease in women. It is estimated that there are approximately 1 billion menopausal women 
by 2050 (6-8). It is, therefore, of considerable significance to research AS in postmenopausal women.

There was no equivalent term as "atherosclerosis" or "atherosclerosis in postmenopausal women" in ancient Chinese medical literature. It falls under the category of "chest bi-impediment" or "cardiac pain." The collateral disease theory is a theoretical system with the characteristics of traditional Chinese medicine, which studies two branches: vessel collateral theory and qi collateral theory $(9,10)$. Vessel collateral system and vascular system are similar in structure and function (11), so vessel collateral theory is often used for vessel collateral problems, including AS. Qi collateral theory is often used for qi collateral problems, including disease of endocrine. A significant increase in the incidence of AS in post-menopausal women is related to estrogen deficiency, which is associated with dysfunction of the qi collaterals (3). Both vessel collateral theory and qi collateral theory play essential roles in the prevention and treatment of AS in postmenopausal women. Previous studies have confirmed that Tongxinluo capsule, under the guidance of collateral disease theory, inhibits the progression of AS through a variety of ways, such as regulating blood lipid, preventing plaque rupture, protecting vascular endothelium, inhibiting inflammation and regulating immune response (12-14). This review focuses on the guiding role of collateral disease theory in postmenopausal AS.

We present the following article in accordance with the Narrative Review Checklist (available at http://dx.doi. org/10.21037/apm-20-1257).

\section{Qi collaterals and vessel collaterals: circulate qi and blood and nourish yin and yang}

Meridians, the pathways of qi and blood circulation, are an essential structural and functional part of the human body. The Ling Shu Mai Du (Chapter 17, Miraculous Pivot) states, "Meridians are in the interior. Their transverse branches are called collaterals. The divergent branches of collateral are called minute collaterals." Collaterals are like a multi-layer network throughout the body. They act to circulate qi and blood and nourish yin and yang. Zhou Xuehai, a Qing Dynasty physician, proposed the concepts of "fine collaterals of qi" and "fine collaterals of blood." In a broad sense, collaterals include the "fine collaterals of qi" that circulate meridian qi and "fine collaterals of blood" that circulate blood (15). The two parts are separate but interconnected. Qi collaterals carry the Yuan-primordial qi, Zong-pectoral qi, Wei-defensive qi, the qi of the zangfu organs, and the qi of meridians and collaterals. Qi that circulates within meridians is called meridian qi, which becomes collateral qi when it enters collateral. Collateral qi then becomes a structural and functional part of zang-fu organs when it enters the zang-fu organs. These functions are highly correlated with the nerve-endocrine-immune system (NEI system) in modern medicine (16).

The Su Wen Mai Yao fing Wei Lun (Chapter 17, Basic Questions) states, "Vessel is the house of blood." Vessel collaterals diverge from blood vessels. The vessel collateral system formed by minute collaterals and their subbranches (17).

The correlation between qi collaterals and vessel collaterals manifests the interactions between qi and blood. Qi and blood are two cornerstone concepts in Chinese medical theory. They both play a significant role in life activities. Qi is yang in nature; it governs moving and warming. Blood is yin in nature; it governs tranquility, moistening, and nourishing. The Nan Fing (The Classic of Difficult Issues) states, "Qi governs warming, and blood governs nourishing," "qi is the commander of blood," and "blood is the mother of qi."

In the vessel collateral system, "qi is the commander of blood" that can be understood as qi collaterals, promoting vessel collaterals and keeping blood circulating within the vessels. The Yuan-primordial qi is the driving force for the normal functioning of zang-fu organs and the entire body. The Zong-pectoral qi governs respiration and distributes clean qi (inhaled through the lungs) all over the body. Qi of the zang-fu organs plays a vital role in regulating blood circulation. "Blood is the mother of qi" can be understood as vessel collaterals, providing nutrients for qi collaterals. The Xue Zheng Lun (Treatise on Blood Syndrome) by Tang Rongchuan, a Qing Dynasty physician, has stated that "blood carries qi and qi circulating blood." The Sheng $f_{i}$ Zong Lu (Comprehensive Recording of Divine Assistance) by Zhao Ji, a Song Dynasty physician, also states, "Qi needs to be carried by the blood, and blood needs to be circulated by qi. The two are inseparable." The Huang Di Nei fing Suwen fi Zhu (Annotations to Basic Questions, Yellow Emperor's Internal Classic) by Zhang Yinan, another Qing Dynasty physician, states, "blood within the vessels permeates through the vessel collaterals, and qi outside the vessels enters the blood through qi collaterals." Collaterals are essential for the normal functioning of qi and blood (Figure 1). 


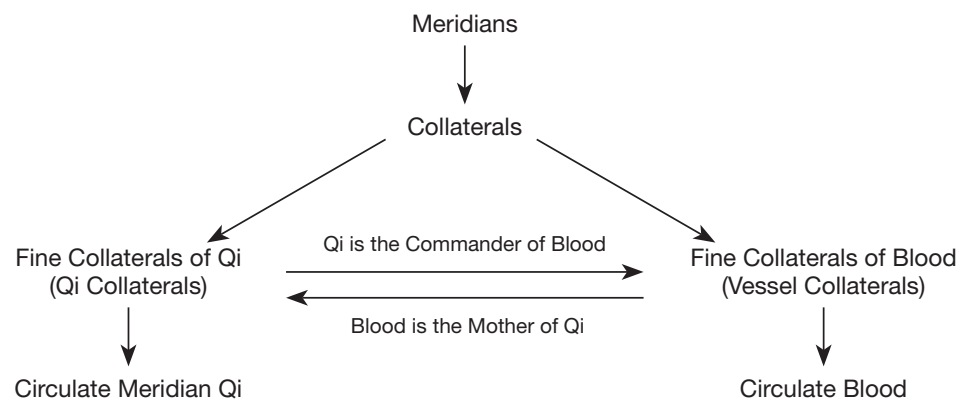

Figure 1 The correlation between qi collaterals and vessel collaterals. "qi is the commander of blood": qi collaterals promoting vessel collaterals and keeping blood circulating within the vessels. "Blood is the mother of qi": vessel collaterals providing nutrients for qi collaterals.

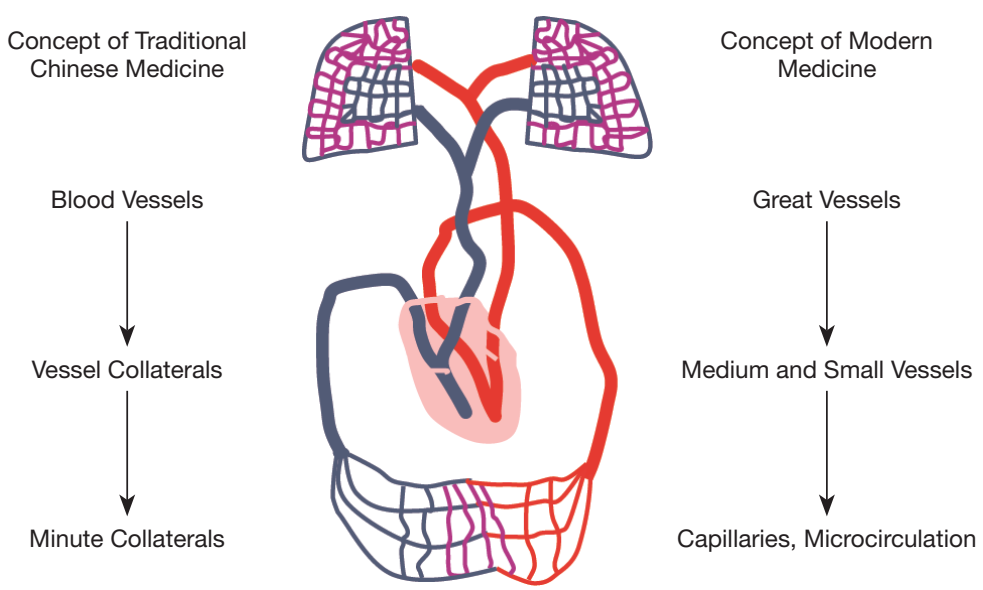

Figure 2 The concept of "vessel collateral-vascular system disease".

\section{AS in postmenopausal women are associated with both vessel collaterals and qi collaterals}

\section{AS: a typical vessel collateral-vascular system disease}

The vessel collateral theory further developed the understanding of "blood vessels" in Huang Di Nei fing (Yellow Emperor's Internal Classic). Blood vessels, vessel collaterals, minute collaterals of the vessel collateral system are anatomically and functionally like arteries, veins, capillaries, and microcirculation in modern medicine (Figure 2). The concept of "vessel collateral-vascular system disease" (11) is on the close correlation between vessel collateral and vascular system. In a narrow sense, vessel collateral-vascular system diseases refer to conditions with functional or structural injury to collaterals. In a broad sense, they include pathogenic factors and later pathological changes of zangfu organs and tissues (9). The "chest bi-impediment" or "cardiac pain" was first mentioned in Huang Di Nei fing (Yellow Emperor's Internal Classic). According to collateral disease theory, this condition is associated with retention of cold, stagnant qi, static blood, and phlegm-fluid in the chest, resulting in collateral obstruction, contracture, or, in severe cases, blockage. The Su Sen Zbi Zhen Yao Da Lun (Chapter 74, Basic Questions) states, "Cold may affect the blood and blood vessels..., causing severe cardiac pain”. The Su Wen fu Tong Lun (Chapter 39, Basic Questions) states, "Retained cold qi outside the vessels may cause the vessels to contract and result in sudden pain." Located in "vessels," AS is a typical "vessel collateral-vascular system disease"

\section{Estrogen deficiency after menopause and functional disorder of qi collaterals}

As mentioned above, AS is a typical "vessel collateral- 
vascular system disease;" however, it is closely associated with "qi." As the functional and structural carrier of qi, qi collaterals connect, regulate, warm, and nourish the body, defend the body against exogenous pathogens, convey messages, and support homeostasis. AS after menopause is associated with functional disorders of qi collateral in connecting the body and supporting homeostasis.

Collaterals connect zang-fu organs, four limbs, five sense organs, and nine orifices into an organic whole and support homeostasis of the body, known as yin-yang balance in Chinese medicine. They are like the endocrine system that regulates the body through releasing chemical substances, including estrogen.

As for the understanding of menstruation in Chinese medicine, the Su Wen Shang Gu Tian Zhen Lun (Chapter 1, Basic Questions) clearly states the close association between Tiangui and menstruation, "With the abundance of Tiangui and flourishing flow of qi and blood in the Ren and Chong meridians, menstruation occurs at the age of 14 . Women with menstruation can get pregnant; with Tiangui exhaustion and qi decline and blood in the Ren and Chong meridians, menopause occurs at the age of 49. Women cannot get pregnant afterward." Wu Kun explained in the Su Wen Wu Zbu (Wu's Annotations to Basic Questions), "The Gui means kidney water..., which is inborn and endowed by heaven (Tian), hence the name "Tiangui." Ma Shi also explained, "Tiangui means yin essence, i.e., kidney water. As kidney water is accumulated from pre-natal (Xian Tian in Chinese) qi, yin essence is called Tiangui." Tiangui is hereditary and transformed from kidney essence and qi (18). Kidney essence governs growth, development, and reproduction. The kidney stores essence and the essence transforms into the blood. With abundant kidney qi and Tiangui, women have menstruation and the ability to get pregnant. After experiencing menstruation, pregnancy, childbirth, and lactation, women gradually age. It is said that half of the yin qi become lost at the age of 40, followed by weight gain, reduced hearing, and weak eyesight at the age of 50. Kidney essence is the substantial foundation of menstruation. Abundant kidney essence guarantees normal menstruation, while exhaustion of kidney essence causes a deficiency of the Ren and Chong meridians. The Fu Qing Zhu Nŭ Ke (Fu Qing-Zhu's Treatise on Gynecology) states, "With kidney qi deficiency, women do not have abundant kidney essence to transform into menstruation." The $Y i$ Xue Zheng Zhuan (Orthodox Introduction to Medicine) states, "Menstrual blood is transformed from kidney water. Exhaustion of kidney water may cause menopause." These results match the modern understanding of the role of the hypothalamic-pituitary-gonadal axis in promoting the development and maturity of reproductive organs, ensuring normal growth, development, and reproduction of organs and tissues, and affecting the aging process (16).

During the peri-menopausal period, women are at risk of developing cardio-cerebrovascular diseases, including arterial plaque, atherosclerotic coronary artery disease, or ischemic stroke $(19,20)$. Contributing factors include ovary failure, decrease in estrogen (E2) levels, absence of negative feedback inhibition, functional disorder of hypothalamicpituitary-gonadal axis, excessive release of gonadotrophinreleasing hormone $(\mathrm{GnRH})$, follicle-stimulating hormone (FSH) and luteinizing hormone (LH) $(21,22)$, along with functional disturbance of multiple endocrine-regulating centers (especially the hypothalamus and pituitary gland) and cessation of estrogen protection on blood vessels. Consequently, AS in postmenopausal women is closely associated with a disorder of the hypothalamic-pituitarygonadal axis and dysfunction of qi collateral in regulating the homeostasis (16).

\section{$A S$ in postmenopausal women are associated with both qi collaterals and vessel collaterals}

The Tiangui is associated with kidney essence, and its essence transforms into qi. The Lei fing (The Classified Classic) states, its essence transforms into qi. The Yuanprimordial qi is transformed from essence." The Yuanprimordial qi is the foundation of all types of qi. The Nan fing (The Classic of Difficult Issues) states, "The origin of Yuan-primordial qi is the foundation of the twelve regular meridians, the stirring qi between the kidneys, the source of the five zang and six fu organs, and the root of the twelve regular meridians..." From adolescence to menopause, kidney essence slowly becomes deficient and does not transform into Yuan-primordial qi, a crucial component of Zong-pectoral qi. The Yi Xue Zhong Zhong Can Xi Lu (Records of Chinese Medicine with Reference to Western Medicine) states, "the Zong-pectoral qi is on Yuan-primordial qi and nurtured by qi transformed from water and grains." The Zong-pectoral qi is separated into Ying-nutritive qi and $W e i$-defensive qi flowing through the heart vessels. Zhang Jingyue, a well-known Ming Dynasty physician mentioned in the Lei fing (the Classified Classic), "Qi in the exterior (Wei-defense) and interior (Ying-nutrients) are connected through collateral." The connection between Yingnutrients and $W e i$-defense provides necessary conditions 

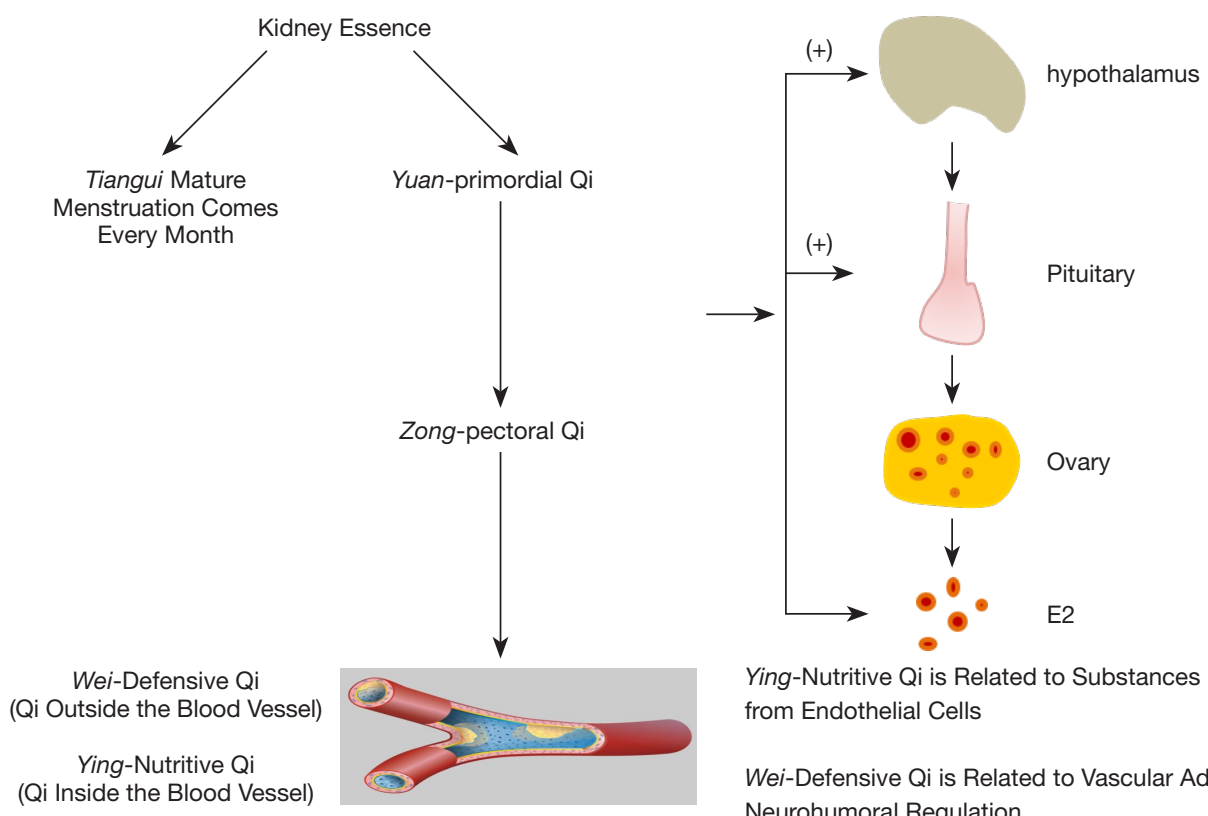

Ying-Nutritive Qi is Related to Substances Released from Endothelial Cells

Wei-Defensive Qi is Related to Vascular Adventitia/ Neurohumoral Regulation

Figure 3 Atherosclerosis in post-menopausal women is associated with both qi collaterals and vessel collaterals.

for metabolism and energy conversion. The Shang Han Lun (Treatise on Cold Damage) states, "Incoordination between Ying-nutrients and Wei-defense causes blood stagnation." Blood stagnation in AS is caused by agerelated kidney essence deficiency, followed by a deficiency of qi and blood, just as the statement in Chen Su'an Fu Ke Bu Fie (Supplementary Annotations to Chen Su'an Gynecology), "Stagnant blood contributes to $70-80 \%$ of retained menses". Another major factor is age-related kidney deficiency, followed by collateral qi deficiency and stagnation.

Analysis of patterns in 3,469 AS cases has shown that collateral qi stagnation and deficiency stagnation are the primary pathology and proceed through the whole process of vessel collateral-vascular system diseases (23-25). Laboratory studies have suggested a correlation between ying-nutritive qi and vasoactive substances released from endothelial cells and between Wei-defensive qi and vascular adventitia/ neurohumoral regulation (26). Collateral qi stagnation or deficiency stagnation may disrupt the homeostatic mechanism of NEI network factors and result in endothelial dysfunction and AS (27). AS in postmenopausal women are associated with kidney essence deficiency. As a vessel collateral disease, it is related to the vascular endothelial and mesangial injury. Simultaneously, it is a qi collateral disease and relates to the dysfunctional hypothalamic-pituitary-gonadal axis and disturbed neurohumoral regulation. Therefore, it is beneficial to understand and treat AS from the collateral disease theory (Figure 3).

\section{The role of collateral disease theory in AS prevention and treatment}

\section{Replenish kidney essence is essential for AS in postmenopausal women}

The fi Sheng Fang (Formula to Aid the Living) states, "Tiangui is the essence, and essence is the foundation of life." The essence is stored in the kidney. Kidney essence insufficiency may cause exhaustion of tiangui, which in turn results in menopause. Therefore, the underlying cause of AS in postmenopausal women is kidney essence deficiency, also, AS is directly associated with blood vessels. Collateral qi deficiency and stagnation is the leading cause of AS, a typical vessel collateral-vascular system disease (27). Since collateral deficiency may cause stagnation, it is essential to replenish kidney essence to keep the collateral unobstructed.

The Lei fing (The Classified Classic) states, "To preserve health, one must replenish its essence. An abundance of essence produces an abundance of qi. An abundance of qi creates a sound mind. A sound mind forms a strong body. And a strong body keeps illnesses away." The Nan fing (The Classic of Difficult Issues) states, "the treatment strategy 
for kidney deficiency is to replenish essence." Most (kidney) essence-replenishing herbs contain phytoestrogens (PEs) and can increase the ovarian response to gonadotropin stimulation, lower FSH levels, and elevate E2 levels (28). Also, they can increase the ovarian weight, induce oocyte maturation and ovulation, and accelerate the functional recovery of the pituitary gland, ovary, and adrenal gland (29). Through analysis of the studies on essence-replenishing herbs, Fan et al. produced the idea that "essence" can be understood as a functional state in a microcosmic and systemic network. In this sense, replenishing essence can regulate the NEI network, coordinate the hypothalamicpituitary-adrenal-gonadal axis, and balance the functional state of the human body (30). What is more, formulas to tonify kidney and replenish essence can regulate the female reproductive axis via multi-targeted organs in multiple levels $(31,32)$.

\section{Distinctive strengths of Bazi Bushen capsule (BZBS) in AS prevention and treatment}

Replenishes kidney essence, coordinates yin and yang, tonifies kidney qi and circulates blood

The concept of "tonifying the kidney" was limited to tonify kidney yin and kidney yang before the Ming and Qing Dynasties (1368-1911). Physicians in the Ming and Qing Dynasties started to realize that kidney essence insufficiency is the underlying cause of kidney deficiency, because kidney qi, kidney yin, and kidney yang are all transformed from kidney essence. Consequently, it is vital to replenish kidney essence to tonify the kidney, supplemented by harmonizing kidney yin and kidney yang, and tonifying kidney qi.

As a typical formula to tonify collateral deficiency and unblock stagnation, the BZBS was modified from the $W u z i$ Yanzong Wan (Five-Seed Progeny Pill) in Yi Xue Ru Men (Introduction to Medicine) by a Ming dynasty physician Li Chan. The five seeds in this formula are Gou Qi Zi (Fructus Lycii), Tu Si Zi (Semen Cuscutae), Fu Peng Zi (Fructus Rubi) and Che Qian Zi (Semen Plantaginis). Our study was the first time a formula to replenish kidney essence using medicinal seeds, or fruits were used. According to the Ben Cao Zheng Yi (Orthodox Interpretation of the Materia Medica), "Medicinal seeds/fruits are hard and substantial. They act to tonify the yin of the five zang-organs and replenish their essence and qi. As they are heavy, medicinal seeds/fruits benefit the kidney and strengthen the kidney qi." Ye Tianshi, a distinguished Qing Dynasty physician, proposed the theory of "replenishing kidney essence with medicinal animals." In his Lin Zheng Zhi Nan Yi An (Case Records as a Guide to Clinical Practice), Ye Tianshi used Lu Rong (Cornu Cervi Pantotrichum), cattle or goat bone marrow, or Lu fiao fiao (Colla Cornus Cervi) to replenish essence and marrow. Although he had no fixed formula, his idea was an inspiration to the combination of medicinal animals into kidney-tonifying formulas.

The BZBS was developed on the above kidneytonifying theory. It consists of eight medicinal seeds: Gou Qi Zi (Fructus Lycii) and Tu Si Zi (Semen Cuscutae) to replenish and benefit kidney essence; $W u W e i Z i$ (Fructus Schisandrae Chinensis), Fu Peng Zi (Fructus Rubi) and fin Ying $\mathrm{Zi}$ (Fructus Rosae Laevigatae) to replenish and secure kidney essence; She Chuang Zi (Fructus Cnidii) and fiu Cai $Z i$ (Semen Allii Tuberosi) to warm yang and benefit essence; and Chuan Lian Zi (Fructus Toosendan), the only one to circulate qi, especially qi of the liver and kidney, and help prevent stagnation from essencereplenishing ingredients. Considering from the theory of "using food to replenish essence" in Huangdi Neijing (Yellow Emperor's Internal Classic) and of "using medicinal animals to replenish essence" by Ye Tianshi, the BZBS contains two ingredients from medicinal animals: Lu Rong (Cornu Cervi Pantotrichum) to "regenerate essence, replenish the marrow, nourish the blood, warm yang, and strengthen the sinews and bones" (recorded in the Ben Cao Gang Mu, i.e., the Compendium of Materia Medica). Hai Ma (sea horse) to tonify the kidney, replenish essence, and harmonize between qi and blood.

Also, the BZBS contains Sheng Di Huang (Radix Rehmanniae) to nourish kidney yin, Yin Yang Huo (Herba Epimedii), Ba fi Tian (Radix Morindae Officinalis) and Rou Cong Rong (Herba Cistanches) to warm kidney yang; Ren Shen (Radix et Rhizoma Ginseng) to tonify Yuan-primordial qi (recorded in the Yi Xue Qi Yuan, i.e., Origins of Medicine) and circulate blood); and Niu Xi (Radix Achyranthis Bidentatae) to tonify the liver and kidney, guide the other ingredients downward, and circulate collateral qi. Of the twelve ingredients, Ren Shen (Radix et Rhizoma Ginseng) and Niu Xi (Radix Achyranthis Bidentatae) supplement qi, tonify the liver and kidney and simultaneously, circulate qi and blood.

\section{Contains PEs and, protects blood vessels}

PEs which is a naturally occurring nonsteroidal plant compound found in plants, fruits, and vegetables. Due to its structural similarity with estradiol, it can cause estrogenic effects but does not cause side effects, including 
endometrial hyperplasia (33). Through the mechanism in lowering lipid, anti-inflammation, anti-oxidative stress, protecting the vascular endothelial function, and inhibiting smooth muscle cell proliferation or migration $(34,35)$, PEs exert an anti-AS effect.

Our previous study found that BZBS contains 11 PEs, namely isoquercitrin, hyperoside, verbascoside, epimedin A, icariin, baohuoside I , imperatorin, osthole, catalpol, deoxyschizandrin, schisandrin B (36). BZBS lowed serum lipid levels, and improved fatty acid metabolism in highfat diet-fed, ovariectomized female $\mathrm{ApoE}^{-/-}$mice (36). Disturbance of lipid metabolism is one of the mechanisms of occurrence and development of AS (37,38), and PEs inhibit the progression of AS by improving lipid metabolism (39). These supply an experimental basis for BZBS treatment of postmenopausal AS.

\section{Conclusions}

Vessel collaterals and qi collaterals are the two main branches of collateral disease theory in Chinese medicine. The former is significant in understanding typical vessel collateral—vascular system diseases like AS, while the latter is significant in understanding nerve-endocrine-immune disorders (10). Both take part in AS in postmenopausal women due to the interaction between qi and blood. For postmenopausal AS, the supplementation of exogenous estrogen is controversial (33). The BZBS acts to replenish kidney essence. Its ingredients have PEs and protect blood vessels. An in-depth study on its role in postmenopausal AS using modern laboratory techniques might offer a new perspective on the choice of new medicine for safe, effective interventions.

\section{Acknowledgments}

Funding: The work was sponsored by the National Key Research and Development Program-Modernization of Traditional Chinese Medicine (Grant number 2017YFC1700501).

\section{Footnote}

Reporting Checklist: The authors have completed the Narrative Review Checklist. Available at http://dx.doi. org/10.21037/apm-20-1257

Conflicts of Interest: All authors have completed the ICMJE uniform disclosure form (available at http://dx.doi. org/10.21037/apm-20-1257). All authors report grants from China National Center for Biotechnology Development during the conduct of the study.

Ethical Statement: The authors are accountable for all aspects of the work in ensuring that questions related to the accuracy or integrity of any part of the work are appropriately investigated and resolved.

Open Access Statement: This is an Open Access article distributed in accordance with the Creative Commons Attribution-NonCommercial-NoDerivs 4.0 International License (CC BY-NC-ND 4.0), which permits the noncommercial replication and distribution of the article with the strict proviso that no changes or edits are made and the original work is properly cited (including links to both the formal publication through the relevant DOI and the license). See: https://creativecommons.org/licenses/by-nc-nd/4.0/.

\section{References}

1. Sima P, Vannucci L, Vetvicka V. Atherosclerosis as autoimmune disease. Ann Transl Med 2018;6:116.

2. Barton M. Cholesterol and atherosclerosis: modulation by oestrogen. Curr Opin Lipidol 2013;24:214-20.

3. Barrett-Connor E, Bush TL. Estrogen and coronary heart disease in women. JAMA 1991;265:1861-7.

4. Muka T, Nano J, Jaspers L, et al. Associations of Steroid Sex Hormones and Sex Hormone-Binding Globulin With the Risk of Type 2 Diabetes in Women: A PopulationBased Cohort Study and Meta-analysis. Diabetes 2017;66:577-86.

5. Boardman HM, Hartley L, Eisinga A, et al. Hormone therapy for preventing cardiovascular disease in postmenopausal women. Cochrane Database Syst Rev 2015:CD002229.

6. Barton M, Meyer MR. Postmenopausal hypertension: mechanisms and therapy. Hypertension 2009;54:11-8.

7. Gurney EP, Nachtigall MJ, Nachtigall LE, et al. The Women's Health Initiative trial and related studies: 10 years later: a clinician's view. J Steroid Biochem Mol Biol 2014;142:4-11.

8. Clarkson TB, Melendez GC, Appt SE. Timing hypothesis for postmenopausal hormone therapy: its origin, current status, and future. Menopause 2013;20:342-53.

9. Wu YL, Yuan GQ, Jia ZH, et al. Academic Status and its Application Value of Vessel Collateral Theory. J Tradit 
Chin Med 2012;53:3-7.

10. Wu YL. Qi Collateral Doctrine--Preface to the Qi Collateral Theory. Chinese Journal of Difficult and Complicated Cases 2018;17:184-6.

11. Wu YL. Construction of Vessel Collateral Theory and Its Guiding Role in the Prevention and Treatment of Vascular Diseases. Chin J Integr Med 2017;37:147-8.

12. Ma J, Qiao L, Meng L, et al. Tongxinluo may stabilize atherosclerotic plaque via multiple mechanisms scanning by genechip. Biomed Pharmacother 2019;113:108767.

13. Chen Y, Li M, Zhang Y, et al. Traditional Chinese medication Tongxinluo attenuates apoptosis in oxLDL-stimulated macrophages by enhancing Beclin1-induced autophagy. Biochem Biophys Res Commun 2018;501:336-42.

14. Chen WQ, Zhong L, Zhang L, et al. Chinese medicine tongxinluo significantly lowers serum lipid levels and stabilizes vulnerable plaques in a rabbit model. J Ethnopharmacol 2009;124:103-10.

15. Wu YL. Exploration on the Correlation bewteen Vessel Collaterals and Vascular System. J Tradit Chin Med 2007;48:5-8.

16. Wu YL. Analysis on the Correlation bewteen Qi Collaterals and NEI Network. J Tradit Chin Med 2005;46:723-6.

17. Wu YL. Collateral theory and vascular lesion treatment. Am J Chin Med 2009;37:241-52.

18. Cheng A. Tiankui Theory in Neijing and its Application in Peri-menopausal Syndrome [Doctor]: Guangzhou University of Traditional Chinese Medicine; 2005.

19. Zhao D, Guallar E, Ouyang P, et al. Endogenous Sex Hormones and Incident Cardiovascular Disease in PostMenopausal Women. J Am Coll Cardiol 2018;71:2555-66.

20. Glisic M, Mujaj B, Rueda-Ochoa OL, et al. Associations of Endogenous Estradiol and Testosterone Levels With Plaque Composition and Risk of Stroke in Subjects With Carotid Atherosclerosis. Circ Res 2018;122:97-105.

21. Djahanbakhch O, Ezzati M, Zosmer A. Reproductive ageing in women. J Pathol 2007;211:219-31.

22. Vadakkadath Meethal S, Atwood CS. The role of hypothalamic-pituitary-gonadal hormones in the normal structure and functioning of the brain. Cell Mol Life Sci 2005;62:257-70.

23. Wei C, Chang LP, Zhao SS. Exploration on the Role of Vessel Collateral Theory in the Prevention and Treatment of "Vessel Collateral--Vascular System Diseases". Chinese Journal of Difficult and Complicated Cases 2015:1101-3.

24. Yuan GQ, Jia ZH, Gao HL, et al. Mathematical Model
Analysis: Effect of Collateral qi deficiency/stagnation on Endothelial Function and Intervention Efficacy of Collateral-Unblocking Medicine. Chinsese Journal of Basic Medicine in Traditional Chinese Medicine 2010;16:1062-6.

25. Jia ZH, Gao HL, Gu CH, et al. Research on Common Pathogenesis of Vessel Diseases of 3469 Patients based on TCM Syndrome Distribution. J Tradit Chin Med 2009;50:920-4.

26. Jia ZH, Zhang QY, Yuan GQ, et al. Study on the Correlations bewteen NEI Network Factor Secretion and Endothelial function with the model of Collateral-qi Deficiency/Stagnation atherosclerosis. J Tradit Chin Med 2010;51:1121-4,30.

27. Wei C, Wu YL, Jia ZH, et al. Balance between YingNutrients and Wei-Defense in the Prevention and Treatment of Vascular Diseases. J Tradit Chin Med 2013;54:110-3.

28. Li XL, Shi CZ, Cui XP. Influence of the Yin-Yang Sequential Therapy following the Menstrual Cycles on the Sexual Functions of Patients with Premature Ovarian Failure. Journal of Shaanxi College of Traditional Chinese Medicine 2012;35:43-6.

29. Li Y, Yang L, Wang JR. Kidney essence-Replenishing Methods for Poor Ovarian Reserve. Journal of Shaanxi College of Traditional Chinese Medicine 2014;37:57-8.

30. Fan Y, Chen LM. Discussions on the Role and Research Thinking of Essence from the Idea that "the Kidney Stores Essence". Chinese Journal of Information on Traditional Chinese Medicine 2002;9:3-4.

31. Shen ZY, Cai DP. Study on the Regulative Rule of Reinforcing Shen Principle on Sexual Precocity and Senescence at the Molecular Level. Chin J Integr Trad West Med 2005;25:549-51.

32. Shen H, Cai DP, Chen BY. Effects of Kidney-tonifying Chinese Herbal Medicine on Hypothalamic-Pituitary Gonadotrophic Function J Chin Integr Med 2004;2:53-7.

33. Schenck-Gustafsson K, Brincat M, Erel CT, et al. EMAS position statement: Managing the menopause in the context of coronary heart disease. Maturitas 2011;68:94-7.

34. Shen R, Zhang CC, Jiang WY, et al. Effect of ICA on White Fat Metabolism in Mice with ApoE Gene Knockout. Acta Univ Tradit Med Sin Pharmacol Shanghai 2016;30:57-60.

35. Hu L, Dong L, Qu L, et al. Effect of Lycium barbarum polysaccharide on Atherosclerois in Mice by Up-regulating Adiponectin Expression. Drug Evaluation Research 2017;40:292-9. 
36. Huang D, Hu H, Chang L, et al. Chinese medicine Bazi Bushen capsule improves lipid metabolism in ovariectomized female ApoE-/- mice. Ann Palliat Med 2020;9:1073-83.

37. Suciu CF, Prete M, Ruscitti P, et al. Oxidized low density lipoproteins: The bridge between atherosclerosis and autoimmunity. Possible implications in accelerated atherosclerosis and for immune intervention in

Cite this article as: Huang D, Jia Z, Chang L, Zhou H, Wu W, Wei C. The role of collateral disease theory in the prevention and treatment of atherosclerosis in post-menopausal women: a narrative review. Ann Palliat Med 2020;9(4):2314-2322. doi: 10.21037/apm-20-1257 autoimmune rheumatic disorders. Autoimmun Rev 2018;17:366-75.

38. Salvayre R, Negre-Salvayre A, Camare C. Oxidative theory of atherosclerosis and antioxidants. Biochimie 2016;125:281-96.

39. Bairey Merz CN, Johnson BD, Braunstein GD, et al. Phytoestrogens and lipoproteins in women. J Clin Endocrinol Metab 2006;91:2209-13. 